\title{
A Novel Security Approach for Communication using IOT
}

\author{
Gowtham M \\ Assistant Professor \\ Department of Computer Science \& Engineering \\ Rajeev Institute of Technology, Hassan \\ Karnataka, India
}

\author{
M. Ramakrishna \\ Professor \& Head \\ Department of Computer Science \& Engineering \\ Vemana Institute of Technology, Bangalore \\ Karnataka, India
}

\begin{abstract}
The Internet of Things (IOT) is the arrangement of physical articles or "things" introduced with equipment, programming, sensors, and framework accessibility, which enables these things to accumulate and exchange data. Here outlining security convention for the Internet of Things, and execution of this relating security convention on the inserted gadgets. This convention will cover the honesty of messages and verification of every customer by giving a productive confirmation component. By this venture the protected correspondence is executed on implanted gadgets.
\end{abstract}

Keywords-Security; SSL; SN; IOT.

\section{INTRODUCTION}

The Internet of Things (IOT) is the arrangement of physical things or "things" embedded with devices, programming, sensors, and framework accessibility, which enables these articles to accumulate and exchange data. The Internet of Things empowers articles to be identified and controlled remotely across over existing framework system, making open entryways for more direct coordination between the physical world and PC based structures, and achieving improved reasonability, precision and cash related ideal position. Everything is strikingly identifiable through its embedded enlisting system however can interoperate inside the present Internet establishment. IOT is required to offer moved system of devices, structures, and organizations that goes past machine-to-machine correspondences (M2M) and spreads a collection of traditions, spaces, and applications. "Things," in the IOT sense, can suggest a wide variety of devices, for instance, heart checking embeds, biochip transponders on estate animals, electric mollusks in coastline waters, automobiles with characteristic sensors, or field operation devices that assistance firefighters in request and spare operations. These contraptions assemble important data with the help of various existing advances and after that independently stream the data between different gadgets.[5][6]

This paper addresses the security issue, by proposing a distributed security convention to fulfill this shifted condition. Secure correspondence is executed on a publicly released stage for the Internet of Things. Finally, the result exhibits that the proposed tradition is gainful to meet the specific goals and significant for the Internet of Things.

\section{LITERATURE SURVEY}

\section{A. "Examine on Security Problems and Key Technologies of} The Internet of Things"

$\mathrm{Xu}$ Xiaohui School of PC, Wuhan University School of money related perspectives and organization, Wuhan University Wuhan, China. 2013. The IOT is an enormous and comprehensively coursed the Internet that things interface things. It partners each one of the articles to the web through information recognizing contraptions. It is the second information wave after Computer, Internet and compact correspondence orchestrate. With the quick progression of the Internet of Things, its security issues have ended up being more idea. This paper addresses the security issues and key progressions in IOT. It clarified the fundamental thoughts and the rule of the IOT and merged the critical characteristics of the IOT and moreover the International essential research results to examination the security issues and key developments of the IOT which remembering the ultimate objective to assumes a positive part in the development and the advancement of the IOT through the exploration.

\section{B. "The Internet ofThings: A survey" Computer Networks, 2010"}

Atzori, Luigi; Iera, Antonio; Morabito, Giacomo, Vol.54 (15), pp.2787-2805 [Peer Reviewed Journal]. This paper addresses the Internet of Things. Fundamental empowering variable of this promising worldview is the reconciliation of a few advancements and interchanges arrangements. Distinguishing proof and following advances, wired and remote sensor and actuator systems, improved correspondence conventions (imparted to the Next Generation Internet), and appropriated insight for savvy items are quite recently the most applicable. As one can without much of a stretch envision, any genuine commitment to the progress of the Internet of Things should essentially be the consequence of synergetic exercises led in various fields of learning, for example, broadcast 
communications, informatics, gadgets and sociology. In such a perplexing situation, this overview is coordinated to the individuals who need to approach this unpredictable train and add to its advancement. Distinctive dreams of this Internet of Things worldview are accounted for and empowering advancements surveyed. What develops is that still significant issues might be confronted by the examination group. The most important among them are tended to in points of interest.

\section{C. "Internet of Things Security Analysis" 2011}

Gan, Gang ; Lu, Zeyong ; Jiang, Jun, International Conference on Internet Technology andApplications, Aug. 2011, pp.1-4

Web of Things is an exceptional data and innovation industry, and in such a situation of the idea and the substance and the expansion of Internet of Things are not extremely unmistakable, the venture of Internet of Things which is a bit of little region with little scale and self-framework acquire satisfying accomplishment and brilliant future, it can advance the improvement of Internet of Things in some degree. In any case, there are some genuine shrouded risk and potential emergency issues. The paper concentrates on the use of Internet of Things in the country and even in the worldwide later on, breaking down the existed security dangers of the Internet of Thing 's arrange focuses, transmission, at long last we propose some suggestive arrangements because of these issues.

\section{D. "Securing IOT for Smart Home System"}

This paper demonstrates an approach to manage combine strong security in passing on Internet of Things (IoT) for astute home structure, together with due idea given to customer convenience in working the system. The IoT splendid home system continues running on common wifi arrange executed in view of the AllJoyn structure, utilizing a hilter kilter Elliptic Curve Cryptography to play out the verifications amid framework operation. A wifi passage is utilized as the inside hub of the framework to play out the framework introductory setup. It is then in charge of confirming the correspondence between the IoT gadgets and in addition giving an intend to the client to setup, get to and control the system through an Android based PDA running legitimate application program.

Security challenges in IOT join assurance, approval and secure end to end affiliation. Security and accommodation are the two noteworthy prerequisites for effective arrangement of IOT in the shrewd home framework in light of Wi-Fi network [6].

\section{PROPOSED SYSTEM}

This paper concentrates on the blueprint of a security tradition for the IOT, and the execution of this contrasting security tradition on the Sensible Things arrange. This tradition won't simply cover the respectability of messages, moreover the affirmation of each customer by giving a capable confirmation segment. It is an average stage for correspondence among sensors and actuators on an overall scale, and empowers an across the board expansion of IOT administrations. This safe correspondence gives a more proficient data transmission component contrasted and one TLS suit correspondence.

The proposed framework fills in as takes after.

1) The client registers and login through an android application.

2) Once the client enrolls the demand is sent to administrator to acknowledge or dismiss the demand. The enrollment procedure gathers information, for example, clients email ID, secret key and the IMEI number of the telephone from which the client is enlisting.

3) After the endorsement of demand from the administrator, the client can login and ask for utilizing the administrations.

4) Once the client asks for an administration the demand is sent to Authority hub, it at that point creates a declaration and sends it to both client and IOT server.

5) The authorization to utilize the administration is allowed as testament; on getting the endorsement the client can pick the vacancy to control the gadget and furthermore the rundown of administrations accessible.

6) The asked information from client is sent to IOT server alongside declaration. The IOT server assesses the authentication gotten from the client application and the expert hub in the event that it coordinates then it permits to control the gadget else the client need to rehash the whole technique.

\section{SECURITY Protocol}

The focal point of the P2P security system for the Internet of Things is the security tradition. This tradition is the base of all structures' correspondence and affirmation. There are two essential parts in this security tradition:

1) Registration

2) Communication

The enlistment procedure is completed between the as of late joined customer and the Specialist Node (SN). There are six sort of messages transmitted amid the primary enlistment handle.

1) Customer to SN: SSL association ask for message, which is to upgrade the security of the accompanying enrollment exchange.

2) Customer to SN: Enlistment ask for message.

3) SN to customer : Registration answer message.

4) Customer to $\mathrm{SN}$ : Authentication marking demand message.

5) $\mathrm{SN}$ to customer: Authentication marking reaction message.

6) Customer to $\mathrm{SN}$ : Verification tolerating message. The detail of these messages could be seen from underneath, 
without the foremost SSL affiliation requests message. A mid the second procedure, correspondence process depends on the main procedure. There are at most five sorts of message, including authentication trade ask for message, declaration trade answer message, session key trade ask for message, session key trade answer message and secure message. More often than not, just secure message is utilized for data transmission.

\section{MOdUles}

In this segment we plate about the modules exhibit in the venture and there depiction

1) Client Authentication

2) Benefit Request

3) Authentication Generation utilizing SHA

4) Authentication Distribution by IOT server

5) Session Management and Request handler

6) Installed C Programming to control equipment

7) Home PC

8) Joining

\section{1) Client Authentication}

In this module the clients downloads the android application and registers by giving the required points of interest such name email-id secret word, the application naturally gets the IMEI number of the enlisting portable number all these data will be put away in database. Once the client enlists, the demand is sent to administrator to endorse or dismiss, once the client login it sidetracks to landing page.

\section{2) Benefit Request}

After the client login he is diverted to landing page where he motivates choices to start the administration a testament from IOT server will be produced and sends a duplicate to both client application and home PC and a session of 120 seconds will be made for client, he needs to control the switches inside the session lapses else he have to login again and ask for administration and get another session.

\section{3) Authentication Generation utilizing SHA}

This model encourages the client to interface with IOT server by issuing testament as an authorization granter. Once the client enroll and ask for administration the AN of IOT server gets client points of interest, for example, IMEI number and an extraordinary ID which is naturally produced to recognize the client. At that point the An utilizations a mix of this two element and creates a remarkable ID, this procedure is finished with the assistance of Hashing calculation.

\section{4) Authentication Distribution by IOT server}

The Authority node and the IOT server integrated together to form this module. Once the user request to control the device, the request is forwarded to IOT server, the IOT server generates a certificate with unique ID to allot the session to the user and sends a copy of certificate to both user and home PC.

\section{5) Session Management and Request handler}

Once the client ask for administration a session of 120 seconds is made for the client. Each time the client controls the change he leaves controller page and each time the session of 120 seconds terminates the client leaves application, he needs to login again and ask for administration and get a session.

The ask for handler gives data about the status of session. On the off chance that the session is dealt with effectively then it advises the achievement status. In the event that the session flops then a message is shown expressing "The home PC is as of now bustling attempt later".

\section{6) Installed C Programming to control equipment}

The equipment part contains an implanted IC circuit or a raspberry $\mathrm{Pi}$ associated with 4 switches which empowers the client to interface numerous gadgets to control. The controlling part is coded in $\mathrm{C}$ programming dialect, the primary capacity of this equipment is, on accepting the flag from IOT server to control the switch, i.e. it should on or off the gadget.

\section{7) Home PC}

The home $\mathrm{PC}$ is associated with the equipment. The serial to USB converter equipment requires drivers that enable the program to speak with equipment. The home PC module gets the authentication from IOT server and sits tight for client order. On getting client summon the equipment controls the switches and the status of switch is shown in the home PC screen. It additionally shows all the data in regards to association. The reset homes PC catch clears all the unexecuted demands from client, we utilize this when the application ends anomalous or when control disappointment happens. On the off chance that we don't reset the PC then the framework tries to execute the old demand whose session has been lapsed.

\section{8) Joining}

In this stage each module is consolidated together and checked if the framework is acting of course with no mistakes in any module after coordination of modules.

\section{ARCHITECTURE}

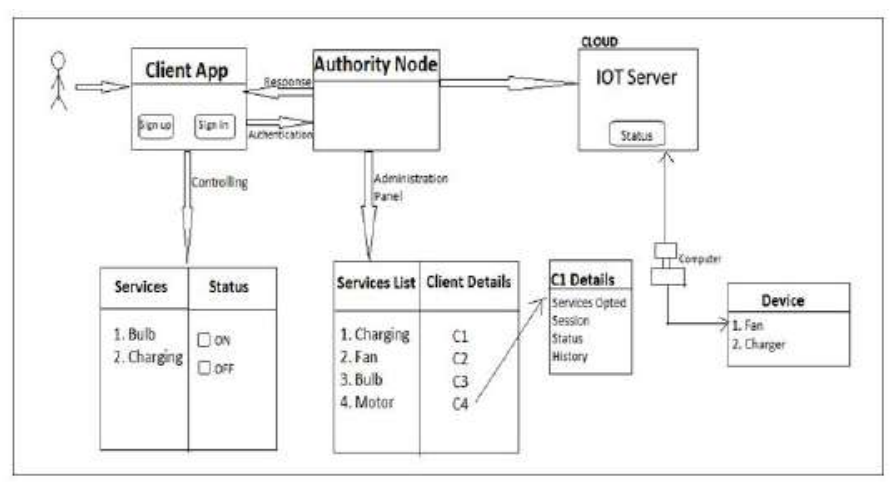

\section{A. Customer(Client App)}

Customer/client needs to enroll to the Authority hub by an Android IOT application. Customer needs to have Android application through which he can control his gadgets remotely. 
These gadgets are associated with PC which is introduced in customer/client home. PC is associated with Internet of Things server.

Customer application gives:

1) Sign up

2) Sign in

When customer is enlisted to $\mathrm{AN}$, it is recognized with declaration (Say one of a kind customer id). Interesting ID alludes to a customer, that he is confirmed to control or screen the gadget.

1) User will get rundown of administrations.

2) Selection of administrations.

3) He can check his administrations status before or in the wake of observing.

\section{B. Specialist NODE(Authority Node)}

1) He keeps up all points of interest of all enlisted/validated customers. Points of interest, for example,

a. Client data (extraordinary id, administrations offered, session).

b. Currently working clients.

c. Client history (time of login, administrations picked, operations done as such for with timings).

2) $\mathrm{AN}$ is associated with IOT server and it keeps up

a. Clients benefit status.

b. Status of PC introduced in home.

\section{Home Personal Computer}

1) Personal PC with IOT programming is associated with IOT server which will continue checking the status of administrations. On the off chance that any adjustment in status PC performs as indicated by it.

2) Devices are associated with PC which is in USB to serial correspondence.

3) Personal PC is associated with equipment unit to which every one of the gadgets can be associated.

\section{RESULT ANALYSIS}

A few Validation checks are performed for "Verified remote exchanging utilizing IOT" application by separating it into segments. Every part has been tried and all test outcomes are working effectively.

The modules in the framework connect as for the determinations and they can be coordinated to create the coveted yield.

It is discovered that the framework fulfills every one of the necessities indicated and delivers the yield with no blunder.

\section{CONCLUSION}

This paper gives an effective security convention to the installed frameworks or stages separating the Internet of Things. Its effectiveness has been fundamentally made strides. As a working research facility model, comparable secure correspondence can likewise be worked for different frameworks. Outfitted with this assurance, individuals' security could be very much ensured in the Internet of Things. This in like manner advances the change of the Internet of Things.

\section{REFERENCES}

[1] Xu Xiaohui School of computer, Wuhan University School of economics and management, "Study on Security Problems and Key Technologies of The Internet of Things" Wuhan University Wuhan, China. 2013.

[2] Atzori, Luigi; Iera, Antonio; Morabito, Giacomo, "The Internet ofThings: A survey" Computer Networks, 2010, Vol.54 (15), pp.27872805 [Peer Reviewed Journal]

[3] Gan, Gang ; Lu, Zeyong ; Jiang, Jun, "Internet of Things Security Analysis" 2011 International Conference on Internet Technology andApplications, Aug. 2011, pp.1-4

[4] Suo, Hui ; Wan, Jiafu ; Zou, Caifeng ; Liu, Jianqi, "Security in the Internet of Things: A Review" 2012 International Conference onComputer Science and Electronics Engineering, March 2012, Vol.3, pp.648-651

[5] Zou, Caifeng, Lu, Zeyong, Morabito, Giacomo, "Access control for IOT devices home automation, of computer science and electronic engineering, jan 2014

[6] Freddy K Santoso, and Nicholas C H VunSchool, "Securing IOT for Smart Home System" of Computer Engineering,Nanyang Technological University, Singapore. 2015

\section{AUTHORS' PROFILE}

Mr. Gowtham M received his B.E. degree in Information Science and Engineering from Kalpataru Institute of Technology, Tiptur. He received M.Tech. degree in Computer Networks and Engineering from National Institute of Engineering, Mysore. He is currently working as Assistant Professor, Department Of CSE at Rajeev Institute of Technology, Hassan, Karnataka, India. His area of interest is Computer Networks, Cloud Computing.

Dr. M Ramakrishna received his B.E. Computer Science and Engineering from Periyar University, Tamilnadu. He received M.E. degree in Computer Science and Engineering from Anna University, Tamilnadu. He received Ph.D. in Computer Science and Engineering from Anna University, Tamilnadu. $\mathrm{He}$ is currently working as Professor \& Head, Department Of CSE at Vemana Institute of Technology, Bangalore, Karnataka, India. His area of interest is Computer Networks. 

distributed under the terms and conditions of the Creative Commons by Attribution (CC-BY) license. (http://creativecommons.org/licenses/by/4.0/). 